TTR

Traduction, terminologie, rédaction

\title{
La souveraineté peut-elle se transférer? Les enseignements de la traduction du traité de Waitangi (1840)
}

\section{René Lemieux}

Volume 29, numéro 2, 2e semestre 2016

Translation and Power: Countertactics

La traduction et le pouvoir : la contre-tactique

URI : https://id.erudit.org/iderudit/1051014ar

DOI : https://doi.org/10.7202/1051014ar

Aller au sommaire du numéro

Éditeur(s)

Association canadienne de traductologie

ISSN

0835-8443 (imprimé)

1708-2188 (numérique)

Découvrir la revue

Citer cet article

Lemieux, R. (2016). La souveraineté peut-elle se transférer? Les enseignements de la traduction du traité de Waitangi (1840). TTR, 29(2), 73-98.

https://doi.org/10.7202/1051014ar

\section{Résumé de l'article}

L'objectif de cet article est d'interroger le concept de souveraineté hérité de la modernité européenne à partir de sa " traduction " en maori dans le traité de Waitangi conclu en 1840 entre les chefs maoris d'Aotearoa (Nouvelle-Zélande) et l'Empire britannique. Le concept de souveraineté est difficilement traduisible en maori puisqu'il ne possède pas d'équivalent direct. Le terme kawanatanga choisi par le missionnaire-traducteur Henry Williams n'est qu'une translittération du mot anglais governor auquel le suffixe -tanga a été ajouté; ce terme rend mal l'idée du pouvoir absolu du souverain. Est-ce une " mauvaise " traduction pour autant? Henry Williams était-il incompétent? A-t-il plutôt voulu sciemment tromper les Maoris, comme le laissent entendre certains chercheurs? Le concept était-il lui-même intraduisible? Lorsqu'on analyse la traduction du terme souveraineté, on découvre qu'il n'y a pas d'équivalence formelle préétablie avant sa réalisation et que la souveraineté ne se transfère pas, mais se performe. Le contenu du concept est ainsi isomorphe à sa production : la souveraineté est une performance, et la traduction comme opération de création de termes participe à son actualisation. En utilisant un terme étranger mais profane pour rendre le concept, Henry Williams, sans peut-être le vouloir ou en être conscient, refuse la souveraineté dans son abstraction et, ce faisant, résiste aux tentatives de sceller l'interprétation du texte dans une unicité souveraine. Vue sous ce nouvel angle, la traduction de Williams participerait d'une " pensée sauvage " au sens de l'anthropologue Pierre Clastres, instituant une relativité toujours vulnérable, mais essentielle dans la perspective d'une traduction postcoloniale.
Ce document est protégé par la loi sur le droit d'auteur. L’utilisation des services d’Érudit (y compris la reproduction) est assujettie à sa politique d'utilisation que vous pouvez consulter en ligne.

https://apropos.erudit.org/fr/usagers/politique-dutilisation/ 


\title{
La souveraineté peut-elle se transférer? Les enseignements de la traduction du traité de Waitangi (1840)
}

\author{
René Lemieux \\ Université de Sherbrooke
}

\section{Résumé}

Lobjectif de cet article est d'interroger le concept de souveraineté hérité de la modernité européenne à partir de sa «traduction» en maori dans le traité de Waitangi conclu en 1840 entre les chefs maoris d'Aotearoa (Nouvelle-Zélande) et l'Empire britannique. Le concept de souveraineté est difficilement traduisible en maori puisqu'il ne possède pas d'équivalent direct. Le terme kawanatanga choisi par le missionnaire-traducteur Henry Williams n'est qu'une translittération du mot anglais governor auquel le suffixe -tanga a été ajouté; ce terme rend mal l'idée du pouvoir absolu du souverain. Est-ce une «mauvaise» traduction pour autant? Henry Williams était-il incompétent? A-t-il plutôt voulu sciemment tromper les Maoris, comme le laissent entendre certains chercheurs? Le concept était-il luimême intraduisible? Lorsqu'on analyse la traduction du terme souveraineté, on découvre qu'il n'y a pas d'équivalence formelle préétablie avant sa réalisation et que la souveraineté ne se transfère pas, mais se performe. Le contenu du concept est ainsi isomorphe à sa production : la souveraineté est une performance, et la traduction comme opération de création de termes participe à son actualisation. En utilisant un terme étranger mais profane pour rendre le concept, Henry Williams, sans peut-être le vouloir ou en être conscient, refuse la souveraineté dans son abstraction et, ce faisant, résiste aux tentatives de sceller l'interprétation du texte dans une unicité souveraine. Vue sous ce nouvel angle, la traduction de Williams participerait d'une «pensée sauvage» au sens de l'anthropologue Pierre Clastres, instituant une relativité toujours vulnérable, mais essentielle dans la perspective d'une traduction postcoloniale.

Mots-clés : traduction des sciences humaines et sociales, colonialisme et impérialisme, Nouvelle-Zélande, langue maorie, traité de Waitangi 


\section{Abstract}

This article questions the concept of sovereignty as European modernity translated it into Māori in the Treaty of Waitangi established in 1840 between the Māori chiefs of Aotearoa (New Zealand) and the British Empire. The concept of sovereignty is difficult to translate into Māori as it has no direct equivalent, and so the term kawanatanga-a transliteration approximating the sound of the English "governor" (kawana + the suffix -tanga) and therefore doing very little to convey the idea of absolute power wielded by a sovereign-was chosen by the missionary and translator Reverend Henry Williams. Did the latter wish to deceive the Māori, as some scholars have suggested? Or was the concept itself simply untranslatable? Careful consideration of this translation problem reveals that no formalized equivalent of "sovereignty" ever precedes its realizationthat "sovereignty," in fact, is not "transferred" at all, but rather performed, its conceptual content and its production in culture isomorphous: sovereignty is performed, and translation, as a concomitant creative operation, partakes in this performance's actualization. By coining a term (kawanatanga) that is at once foreign yet still vernacular, Henry Williams rejects (perhaps without meaning to) "sovereignty" as a ready-made abstraction or transferable signified. And in so doing, he resists the urge to close the text within a univocal interpretation of "sovereignty." Seen from this new perspective, William's translation demonstrates something of anthropologist Pierre Clastres' "pensée sauvage," introduces a potentially vulnerable yet essential relativity into discourse on postcolonial translation.

Keywords: translation in humanities and social sciences, colonialism and imperialism, New Zealand, Māori language, Waitangi Treaty

Au commencement, tout le monde était comme une Amérique.

$$
\text { (Locke, } 1992 \text { [1795], II.5 §49) }
$$

L'acte traductif est bien souvent un acte de création : des concepts doivent parfois être créés pour que le lectorat cible puisse avoir accès à un message. Qu'en est-il des concepts politiques existant dans une société colonisatrice lorsqu'ils sont traduits dans la langue d'une société colonisée? Entre des cultures très éloignées, la création est souvent affaire de rééquilibrage : un équivalent est imposé par la transformation implicite de la signification d'un terme de la langue d'arrivée dans son rapport au concept de la langue de départ :

Translation has been a tool to make what has been conceptualised as incommensurate commensurate-not through the artistry of the translator, the way a gifted 
teacher can communicate a complicated idea to a student without distortion, but rather the way in which a square peg can be made to fit into a round hole if it is pushed and filed enough. (Price et Lugones, 2003, p. 7) ${ }^{1}$

Lobjet de la présente étude, le traité de Waitangi intervenu en 1840 entre l'Empire britannique et les Maoris dans ce qui deviendra la Nouvelle-Zélande, pose le problème de l'incommensurabilité de manière particulièrement intéressante. À ma connaissance, il s'agit d'un cas unique à son époque d'un traité bilingue qui met côte à côte la langue de la société colonisatrice (l'anglais) et celle de la société colonisée (le maori), à tout le moins avant l'établissement du système politique international tel qu'on le connaît de nos jours ${ }^{2}$. Quoique l'ébauche du texte du traité ait d'abord été rédigée en anglais puis traduite en maori, c'est la version en maori qui est signée. De plus, cette version a longtemps été considérée comme la seule version officielle, tant par les autorités coloniales que par les chefs maoris signataires. La version anglaise qui nous est restée est en fait datée du jour de la signature de la version maorie. Quoi qu'il en soit, on considère généralement la version maorie comme une traduction et on l'analyse comme une suite de problèmes de traduction. Un de ces problèmes est la traduction du concept de souveraineté; il s'agit à la fois d'un problème exemplaire et paradigmatique puisque, d'une version à l'autre, le concept ne s'entend pas de la même manière. Cette différence est si grande que l'on considère aujourd'hui en Nouvelle-Zélande qu'il y a deux textes parallèles - the Treaty

1. Price et Lugones se basent en partie sur les travaux de Lydia H. Liu, qui propose une théorie historiciste des équivalences en traduction : « $[\mathrm{A}]$ ny linkages that exist derive from historical coincidences whose meanings are contingent on the politics of translingual practice. Once such linkages are established, a text becomes "translatable" in the ordinary sense of the word.» (Liu, 1995, p. 8) 2. On situe généralement létablissement définitif de ce système basé sur la souveraineté de l'État vers la fin du XIX e siècle (voir Morin, 1997). Le système juridique international qui se construit peu à peu durant le $\mathrm{XX}^{\mathrm{e}}$ siècle obligera à penser la traduction des concepts juridiques d'abord dans les langues européennes (par exemple, lors de la création de la Société des Nations en 1919), puis dans les langues non européennes (lors de la création de l'ONU, qui demande la traduction en chinois et en arabe). La problématique de la traduction en langues locales, minoritaires ou autochtones est aujourd'hui un champ d'étude prolifique du droit comparé (voir Parent, 2009; Anker, 2014b; Geslin, 2015). La traduction du traité de Waitangi en maori fait figure de précurseur de cette problématique. 
et te Tiriti - plutôt qu'un original et sa traduction. Alors qu'un traité en droit devrait être la forme ultime d'une reconnaissance réciproque au sens où chacune des parties reconnaît l'identité de l'autre, le caractère bilingue du traité de Waitangi, unique dans les annales de l'Empire britannique, a plutôt créé un conflit d'interprétation ${ }^{3}$.

Les problèmes de traduction du traité de Waitangi ont donné lieu à au moins trois interprétations différentes. La première, qu'on pourrait qualifier de "populaire», est simple : l'absence d'équivalence entre la version anglaise et la version maorie serait due à une méconnaissance de la langue maorie de la part du missionnaire-traducteur, le révérend Henry Williams (voir notamment Biggs, 1989) ${ }^{4}$. Selon la deuxième interprétation, le traducteur aurait plutôt eu l'intention implicite de tromper les Maoris afin qu'ils signent le traité - dans sa version maorie - sans connaître véritablement le contenu du document qui allait avoir force de loi, à savoir la version "originale» anglaise. C'est l'interprétation privilégiée de nos jours par les chercheurs qui travaillent sur le traité (Fenton et Moon, 2002; SchulteTenckhoff, 2004). La troisième interprétation, proposée par le juriste Richard Dawson, voit plutôt dans la multiplicité des versions l'occasion de développer une herméneutique du droit à travers une nouvelle image de la traduction ${ }^{5}$. Les deux dernières interprétations supposent ainsi, quoi que de manières fort différentes, que la «mauvaise » traduction était intentionnelle, soit parce qu'elle visait à tromper les chefs maoris, soit parce que c'est l'image contemporaine de la traduction comme une mécanique de transfert de sens qui nous oblige, a posteriori, à voir des problèmes

3. Sur le plan juridique, pour éviter le problème du document double, la Cour d'appel de Nouvelle-Zélande a créé les «principes du traité de Waitangi», évitant ainsi de se référer au texte même. On cite généralement quatre de ces principes : «the right of the Crown to govern and the right of Maori to continue to exercise self-determination; on-going partnership with obligations to act reasonably and in good faith; duty to remedy past breaches; active protection " (Court of Appeal of New Zealand, 1987, p. 663 et p. 702-703).

4. Le téléfilm Waitangi: What Really Happened semble perpétuer ce point de vue (Burger, 2011).

5. Dawson (2005) critique la traduction comme métaphore d'un transfert spatial et favorise, ce faisant, un renouvellement de l'ordre juridique néozélandais à partir du concept de fédération appliqué aux souverainetés plurielles. 
là où il n'y en avait peut-être pas à l'origine ${ }^{6}$.

Mon objectif est de développer la troisième interprétation, celle de Dawson, pour la mettre à l'épreuve du concept de souveraineté en montrant que les effets de la traduction aujourd'hui ne pouvaient être connus du traducteur de l'époque. En effet, le problème du transfert du concept de souveraineté est en soi un problème qui relève de la conception de la traduction dans une société coloniale. Dans une telle société, la traduction des concepts juridiques et politiques se produit dans une seule direction : des langues européennes vers les langues autochtones ${ }^{7}$. Je propose de penser la traduction de la souveraineté comme la conséquence d'une souveraineté de la traduction, avec tout ce que le concept de souveraineté suppose : l'imposition d'une interprétation unique, idéale (ou conceptuelle), dont la multiplicité des versions n'est que l'image résiduelle ou accessoire. Il s'agit d'une invitation à repenser la traduction comme pratique sociale à partir de ses conséquences juridico-politiques.

Je débuterai en présentant les grandes lignes du concept de souveraineté et des modalités de son «transfert». Je donnerai ensuite un aperçu de l'histoire du cas précis de la NouvelleZélande en expliquant les points problématiques de la traduction de Williams débattus par les commentateurs. Je terminerai en présentant quelques éléments qui pourraient faire l'objet de futures études sur la traduction dans un contexte colonial. Mon objectif est de montrer que, bien souvent, la traduction et la politique vont de pair, qu'une réflexion sur la traduction peut déboucher sur de nouvelles pratiques politiques, dans l'espoir que ces pratiques réconciliatrices servent à revoir le rôle de la traduction dans les sociétés coloniales.

6. Dawson repère chez le philosophe de la souveraineté Thomas Hobbes l'origine de cette mécanique (Dawson, 2005, p. 34; voir Hobbes, 2002 [1651], Livre I, chap. 4 «De la parole»). On pourrait également voir chez Jakobson (1986) et son analyse de la traduction (inspirée de la cybernétique) l'actualisation contemporaine de cette image mécanique du transfert de sens (ce que je propose dans Lemieux, 2014).

7. On pourrait ajouter les concepts religieux. La traduction qui se fait dans le sens inverse, des langues autochtones vers les langues européennes, est souvent à caractère esthétique ou culturel, exécutée pour un marché littéraire ou savant (traduction de mythes et de légendes conçus comme des récits dénués de tout caractère juridique ou politique). Une étude sur cette directionalité de la traduction en contexte colonial - ce qu'il importe de traduire, à quel moment et pour quelles fins - reste à faire. 


\section{Le concept de souveraineté}

L'origine précise du concept de souveraineté est difficile à situer, car la souveraineté est le produit à la fois de la pensée de théoriciens du droit et de facteurs sociohistoriques. On s'entend toutefois pour voir dans la Renaissance l'époque où s'élabore la forme politique à laquelle la souveraineté donne naissance, l'Étatnation ${ }^{8}$. La course pour les conquêtes du «Nouveau Monde» a partie liée avec des juristes européens qui n'ont jamais mis les pieds hors de leur continent. La chose est importante, car, bien souvent, lorsque des États européens entreprendront de prendre possession de nouvelles terres, ce sera au nom de la «doctrine de la découverte» (Cheyfitz, 1997, p. xiv) et de son corollaire, la terra nullius, qui qualifie un territoire sans souverain identifiable.

Si déjà pour le juriste français Jean Bodin (1530-1596), premier théoricien du concept, la souveraineté est le pouvoir absolu de faire et de défaire les lois (Bodin, 1993 [1583], livre I, chap. X), on tient généralement Thomas Hobbes (1588-1679) pour celui qui institue au concept son fondement, notamment dans son livre Le Léviathan. Il n'est pas anodin que Hobbes donne pour image du concept le nom de ce monstre marin mentionné dans le livre de Job. Lédition princeps du livre le représente : dans la moitié supérieure de la page couverture, on peut voir l'allégorie du monstre sous la forme d'un roi géant. Lorsqu'on y regarde de plus près, on constate que le roi est composé de la multitude du peuple : les individus minuscules, vus de dos, sont disposés en assemblée, ce qui donne l'impression qu'ils sont les maillons d'une cotte que revêt le souverain. Dans la moitié inférieure de la page couverture sont dépeints les deux types d'attributs du monstre, celui de la guerre et du pouvoir temporel à gauche (un fort, une couronne, un canon, etc.), celui de l'Église et du pouvoir spirituel à droite (une église, une mitre, des éclairs, etc.). Une citation du livre de Job y est aussi inscrite : "Non est potestas Super Terram quae Comparetur ei»".

8. On peut situer le changement ailleurs, à partir d'autres paramètres, par exemple, l'élaboration du paradigme scientifique dans l'Angleterre du $\mathrm{XVII}^{\mathrm{e}}$ siècle (voir Bennett, 2007 à propos des conséquences sur le discours des sciences humaines et sociales et leur traduction). Quoi qu'il en soit, le milieu du $\mathrm{XIX}^{\mathrm{e}}$ siècle représente un moment charnière dans ce changement de paradigme qu'il est possible de constater dans la longue durée.

9. Job 41:24 dans la numérotation de la Vulgate, et 41:33 dans les versions modernes. Lemaistre de Sacy traduit : «Il n'y a point de puissance sur la terre qui puisse lui être comparée, [puisqu'il a été créé pour ne rien craindre].» 
Hobbes n'est pas seulement à l'origine du concept de souveraineté : toute sa conceptualisation vise en fait à comprendre l'avènement du concept, qu'il situe dans un contrat entre les membres de la société, chacun prenant conscience que la délégation de son pouvoir individuel dans les mains du souverain assure à l'ensemble la paix sociale et la sécurité de tous. C'est bien la multitude qui crée le monstre qui agit ainsi par désir populaire. La multitude se nie au profit d'une unité transcendantale: l'Un. La souveraineté du corps politique est l'absolutisation des volontés particulières de chacun au profit d'une seule entité dont la parole a force de loi, et sa forme contemporaine est l'État. En conceptualisant un tel mécanisme d'accumulation de pouvoir, Hobbes crée en retour un imaginaire du monde avant l'État, celui de l'état de nature. Il s'agit d'un monde anarchique, un monde où chacun est en guerre contre tous (bellum omnium contra omnes), où l'homme est un loup pour l'homme (bomo homini lupus est) :

Dans un tel état, il n'y a aucune place pour une activité laborieuse, parce que son fruit est incertain; et par conséquent aucune culture de la terre, aucune navigation, aucun usage de marchandises importées par mer, aucune construction convenable, aucun engin pour déplacer ou soulever des choses telles qu'elles requièrent beaucoup de force; aucune connaissance de la surface de la terre, aucune mesure du temps; pas d'arts, pas de lettres, pas de société, et, ce qui est le pire de tout, la crainte permanente, et le danger de mort violente; et la vie de l'homme est solitaire, indigente, dégoûtante, animale et brève. (Hobbes, 2002 [1651], Livre I, chap. 13, p. 108-109)

C'est l'image que les Européens du XVI e siècle ont des sociétés sans État (Clastres, 1974), une image qui perdure bien souvent jusqu'à nos jours (Henderson, 2000). Comme pour les théoriciens politiques subséquents (Locke, 1992 [1795]; Hegel, 1965), un monde sans État - ou simplement situé à l'extérieur de l'Europe - est un monde primitif que la Raison a contourné. Locke, en affirmant qu'à l'origine tout le monde était l'Amérique (1992 [1795], II.5 §49), ne dit pas autre chose; dans son cas, c'est parce qu'il définit l'Amérique par rapport à un manque, celui de l'argent. Lorsqu'on ne reconnaît pas chez l'autre ce qu'on possède chez soi, on attribue à cette différence une défaillance, une défectuosité à laquelle il faut suppléer. 
Notons pour terminer cette courte explication de la souveraineté que son établissement, dans la logique de Hobbes, nécessite une imposition verticale du pouvoir: le souverain est maître de l'interprétation des lois; il les dicte, mais il s'assure également que son interprétation est la seule admissible :

Le pouvoir du souverain ne peut pas, sans son consentement, être transmis à un autre, on ne peut lui confisquer ce pouvoir, il ne peut être accusé par l'un de ses sujets d'avoir commis un tort, il ne peut pas être puni par eux, il est juge de ce qui est nécessaire à la paix, et il est juge des doctrines. Il est l'unique législateur, et le juge suprême des disputes, des moments et des opportunités de la guerre et de la paix. (Hobbes, 2002 [1651], p. 32, Livre II, chap. 20)

Or, la souveraineté se constitue également dans une reconnaissance réciproque des États; elle découpe le monde en territoires, et chacun de ces territoires aura son souverain. La question spécifique au traité de Waitangi est de savoir si les sociétés en dehors de l'Europe, en particulier les sociétés autochtones, sont souveraines et, si c'est le cas, si elles peuvent céder leur souveraineté à un État. Ce problème politique introduit un deuxième questionnement que les commentateurs du traité ne cessent de poser : ces sociétés possèdent-elles le langage nécessaire pour formuler le concept de souveraineté?

\section{Le traité de Waitangi et ses problèmes de traduction}

Le peuplement du pays que l'on nomme la Nouvelle-Zélande a commencé aux alentours du XIII ${ }^{e}$ siècle par des navigateurs polynésiens. L'arrivée des colons britanniques (nommés Pakehas en maori) commence après la cartographie des îles par le capitaine James Cook en 1769 et 1770. Dans les années 1830, alors que les îles sont officieusement sous le contrôle du gouverneur de la Nouvelle-Galles-du-Sud (créée en 1788; aujourd'hui un État australien), les visites de navires français et le projet excentrique de l'aventurier français Charles Philippe Hippolyte, baron de Thierry, de se proclamer roi de la Nouvelle-Zélande (Fenton et Moon, 2002a, p. 55) forcent l'Empire britannique à s'assurer de son contrôle effectif sur l'archipel. Pour contrer le possible projet d'annexion du pays à la France, le Royaume-Uni dépêche en 1835 un Resident - un représentant de l'Empire sans pouvoir exécutif -, James Busby. Avec l'aide d'Henry Williams, missionnaire de la 
Church Missionary Society qui se trouvait lui-même sur les lieux depuis 1823, Busby convainc les tribus (iwi) maories de proclamer l'indépendance de la Nouvelle-Zélande (sous le nom de United Tribes of New Zealand ou, en maori, Wakaminenga o nga Hapu o $\mathrm{Nu}$ Tirene). Une déclaration d'indépendance est rédigée et traduite en maori par Henry Williams, qui avait contribué, en 1827, à une traduction partielle de la Bible en maori ${ }^{10}$.

Cinq ans après la déclaration d'indépendance, l'Empire souhaite renforcer son pouvoir sur l'archipel par la signature d'un traité. Les Maoris y voient de leur côté une garantie juridique pour mieux contrôler l'arrivée de plus en plus grande de colons (Fenton et Moon, 2002b, p. 29) et un moyen d'augmenter le commerce avec les puissances étrangères (Orange, 2010 [1987], p. 46). Au moment où est signé le traité, un courant humaniste est bien implanté au Royaume-Uni, favorisé par la Aborigines' Protection Society fondée en $1836^{11}$. Cette société incite les États à revoir leur conception de l'acquisition de nouvelles colonies (Fenton et Moon, 2002b, p. 27-28). Selon la doctrine de cette société, tout peuple possède des droits naturels comprenant :

1. Their rights as an independent nation. That no country or people has a right by force or fraud to assume the sovereignty over any other nation;

2. That such sovereignty can only be justly obtained by fair treaty, and with their consent. (Aborigines' Protection Society, 1840, p. 14; cité dans Asch, 2014, p. ix)

Cette nouvelle approche convainc l'Empire d'envoyer le capitaine William Hobson en Nouvelle-Zélande pour qu'il signe un traité avec les Maoris confirmant l'établissement de la colonie ${ }^{12}$. Hobson arrive le 29 janvier 1840 dans la baie des Îles où se trouve le village de Waitangi. Lordre reçu de son supérieur, le secrétaire d'État aux colonies, lord Constantine Henry Phipps, $1^{\text {er }}$ marquis de

10. D'autres parties de la Bible seront par la suite traduites par les révérends Robert Maunsell et William Williams (le frère d'Henry) et publiées en 1833 et en 1840. La traduction complète de la Bible est achevée en 1857 et elle sera publiée en 1868, un an après la mort d'Henry Williams (Rogers, 1973). La dernière impression de la Bible en maori, parue en 2013, reprend la version de 1868 avec des modifications mineures (Te Paipera Tapu 1868, 2013).

11. Cette association se situe dans la continuité de la Anti-Slavery Society, qui avait réussi à faire abolir l'esclavage en 1833 au Royaume-Uni.

12. Hobson deviendra le premier gouverneur général de la colonie. 
Normanby, est d'assurer la cession de la souveraineté des Maoris à l'Empire britannique par l'entremise de la signature d'un traité, cette signature étant «conducted on the principles of sincerity, justice, and good faith» (Normanby, 1990 [1839], p. 271; cité dans Fenton et Moon, 2002b, p. 29).

Avec l'aide de son secrétaire James Stuart Freeman et du Resident Busby, Hobson entreprend la rédaction du traité le 3 février 1840. De nombreuses ébauches sont réalisées, mais elles n'ont pas toutes été conservées, à l'exception notable de la version «Littlewood» ${ }^{13}$. Le 4 février à 16 heures, le missionnaire Henry Williams reçoit la version anglaise définitive. Il a l'ordre de la traduire en maori avant la signature, prévue pour 10 heures le lendemain. Il traduit le traité en une nuit avec l'aide de son fils Edward, âgé de 22 ans. Le 5 février, le traité est présenté et expliqué aux chefs maoris réunis à Waitangi. Le traité est discuté toute la journée, et ce n'est que le jour suivant, le 6 février, qu'il est signé par les chefs à Waitangi. A près chaque signature, Hobson se tourne vers le chef qui vient de l'apposer et déclare en maori «He iwi kotahi tātou», parfois traduit par «nous sommes maintenant un seul peuple ${ }^{14}$. Le traité est ensuite transporté autour de l'île du Nord pour être signé par d'autres chefs. Au total, plus de 500 chefs le signeront. Quatre copies du traité sont envoyées aux supérieurs de Hobson, et la traduction originale, qui comporte les signatures, est conservée à Auckland dans un coffre d'acier. Cette traduction a bien failli être détruite en 1841 dans l'incendie du bâtiment où elle était entreposée. Elle est par la suite transférée à Wellington lorsque cette ville devient la capitale du pays en 1865 . La traduction originale est ensuite perdue de vue jusqu'en 1908, année où l'historien Thomas Hocken la retrouve dans le sous-sol

13. Découverte en 1989 par les descendants de Henry Littlewood, témoin pakeha de la signature du traité, et rendue publique le 23 septembre 1992, la version Littlewood est datée du 4 février 1840. Elle diffère légèrement de celle conservée par le gouvernement, datée du 6 février (Doutré, 2005; Loveridge, 2006; Parkinson, 2006). Je n'entrerai pas dans les détails de cette histoire controversée; notons seulement que la difficulté de l'interprétation du traité de Waitangi n'est pas seulement liée à la présence de deux versions en deux langues, mais aussi à celle d'une multiplicité de versions dans chacune des langues.

14. Cette interprétation de la phrase de Hobson est réfutée par plusieurs; la traduction plus exacte serait «nous sommes maintenant un seul esprit», et sa signification serait plutôt «nous nous sommes maintenant entendus» ou «nous sommes maintenant parvenus à un accord» (voir Nairn, 2013). 
d'un des édifices du gouvernement. Elle est maintenant conservée à la Bibliothèque nationale de Nouvelle-Zélande, à Wellington, où il est possible de la consulter.

Du point de vue politique, le traité de Waitangi commence à perdre son statut de document constitutionnel dans les années 1860, à une époque fortement marquée par le racisme de la société majoritaire envers les Maoris (Schulte-Tenckhoff, 2004, p. 108). On prend alors conscience des différences sémantiques entre les versions anglaise et maorie, et, considérant la version anglaise comme étant la version originale, on commande en 1869 une retraduction en maori pour pallier les différences d'interprétation (Fenton et Moon, 2002b, p. 40; pour le texte de cette retraduction, voir Orange, 2010 [1987], p. 257-261). En 1877, le traité est tout simplement annulé par James Prendergast, juge en chef de la Cour suprême de Nouvelle-Zélande (Supreme Court of New Zealand, 1877) ${ }^{15}$. Dans ce jugement, Prendergast affirme que le traité est sans valeur puisqu'il a été signé par des barbares primitifs ("primitive barbarians»). Ce jugement renverse de fait le statut de la version maorie du traité puisque, depuis Hobson, la version signée, celle en maori, était considérée comme le véritable traité. Près de cent ans plus tard, en 1975, une loi est adoptée pour garantir le caractère juridique du traité et établir le Tribunal de Waitangi (New Zealand Parliament/Pāremata Aotearoa, 1975).

Le texte du traité de Waitangi est très court : il se compose de trois articles, d'un préambule et d'une péroraison. Plusieurs critiques ont été formulées concernant la traduction en maori. Pour les commentateurs, chacun des articles pose un problème de traduction particulier. Les principaux problèmes de traduction soulevés dans la littérature sont présentés dans les paragraphes qui suivent.

Dans la version anglaise, le premier article stipule que les chefs cèdent à la Couronne «tous les droits et pouvoirs de souveraineté»(Treaty Times Thirty, 2016, p. 41) ${ }^{16}$. Ce concept

15. À l'époque, la Cour suprême de Nouvelle-Zélande était une cour supérieure, la plus haute instance étant le Comité judiciaire du Conseil privé à Londres. Elle a changé de nom en 1980 (Haute Cour de justice) en vue de la création d'une cour de dernière instance en sol néo-zélandais, la nouvelle Cour suprême en 2003.

16. J'utilise ici la traduction française réalisée par Andrea Sisk King, Daphne Lawless, Eric Savarit, Michèle Ryan et Sandrine Savarit, et révisée par Catherine Sumpter. 
de souveraineté est de loin celui qui cause le plus de problèmes. L'article, reproduit ci-dessous dans la version anglaise datée du 6 février 1840, dans la version maorie réalisée par Henry Williams et dans la rétrotraduction en anglais de sir Ian Hugh Kāwharu (1989), se lit comme suit :

\section{Version anglaise datée du 6 février 1840}

The Chiefs of the Confederation of the United Tribes of New Zealand and the separate and independent Chiefs who have not become members of the Confederation cede to Her Majesty the Queen of England absolutely and without reservation all the rights and powers of Sovereignty which the said Confederation or Individual Chiefs respectively exercise or possess, or may be supposed to exercise or to possess over their respective Territories as the sole sovereigns thereof.

\section{Traduction maorie de Henry Williams}

Ko nga Rangatira o te wakaminenga me nga Rangatira katoa hoki ki hai i uru ki taua wakaminenga ka tuku rawa atu ki te Kuini o Ingarani ake tonu atu - te Kawanatanga katoa o o ratou wenua.

\section{Rétrotraduction de sir Ian Hugh Kāwharu}

The chiefs of the Confederation and all the chiefs who have not joined that Confederation give absolutely to the Queen of England for ever the complete government over their land.

Pour plusieurs commentateurs, la traduction du segment «all the rights and powers of Sovereignty" par «te kawanatanga katoa» ne rend pas bien l'importance du concept de souveraineté à l'époque :

[I]n 1840 legal English, "sovereignty" meant the absolute and indivisible power to legislate, judge, and interpret the law; the absolute power to administer it, and to back up its requirements by force; the sole power to engage in foreign relations and thus to appoint and control diplomats and force of arms. (Sharp, 1990, p. 17; cité dans Dawson, 2005, p. 52)

Le terme kawanatanga rend mal le concept de souveraineté parce qu'il relève de la simple gouvernance ou de l'administration. Il est formé du terme kawana, qui correspond à l'anglais governor, auquel le suffixe -tanga a été ajouté; ce suffixe désigne le caractère 
d'une personne, d'une chose ou d'un concept. Les Maoris connaissaient déjà le terme kawana, car on le trouve dans la Bible traduite par Henry Williams : «[Kawana] had first appeared in early translations of the Bible, where Pontius Pilate was described as a kawana (Matthew 27:1-26)» (Fenton et Moon, 2002a, p. 58). En lisant ce terme dans le premier article du traité, les chefs maoris pouvaient aussi y voir une référence au gouverneur en poste en Nouvelle-Galles-du-Sud, kawana désignant simplement le titre d'un agent officiel britannique subalterne (Laurie, 2002, p.255). Dans les deux cas, le terme indique une subordination plutôt qu'une supériorité politique. Avec le premier article, dans la version qu'ils ont signée, les chefs maoris accordaient à la reine le droit de gouverner leur terre, mais pas nécessairement un droit absolu à une souveraineté pleine et entière.

Dans le deuxième article, les chefs accordent à la Couronne «le droit exclusif de préemption» sur leurs terres tout en garantissant aux chefs et aux tribus, à leurs familles et aux individus, «la pleine possession exclusive et paisible de leurs terres et des domaines, forêts, pêcheries et autres biens» (Treaty Times Thirty, 2016, p. 41). L'article se lit comme suit :

\section{Version anglaise datée du 6 février 1840}

Her Majesty the Queen of England confirms and guarantees to the Chiefs and Tribes of New Zealand and to the respective families and individuals thereof the full exclusive and undisturbed possession of their Lands and Estates Forests Fisheries and other properties which they may collectively or individually possess so long as it is their wish and desire to retain the same in their possession; but the Chiefs of the United Tribes and the individual Chiefs yield to Her Majesty the exclusive right of Preemption over such lands as the proprietors thereof may be disposed to alienate at such prices as may be agreed upon between the respective Proprietors and persons appointed by Her Majesty to treat with them in that behalf.

\section{Traduction maorie de Henry Williams}

Ko te Kuini o Ingarani ka wakarite ka wakaae ki nga Rangitira ki nga hapu - ki nga tangata katoa o Nu Tirani te tino rangatiratanga o o ratou wenua o ratou kainga me o ratou taonga katoa. Otiia ko nga Rangatira o te wakaminenga me nga Rangatira katoa atu ka tuku ki te Kuini te hokonga o era wahi wenua e pai ai te tangata nona 
te Wenua - ki te ritenga o te utu e wakaritea ai e ratou ko te kai hoko e meatia nei e te Kuini hei kai hoko mona.

\section{Rétrotraduction de sir Ian Hugh Kāwharu}

The Queen of England agrees to protect the chiefs, the subtribes and all the people of New Zealand in the unqualified exercise of their chieftainship over their lands, villages and all their treasures. But on the other hand the chiefs of the Confederation and all the chiefs will sell land to the Queen at a price agreed to by the person owning it and by the person buying it (the latter being) appointed by the Queen as her purchase agent.

Alors qu'en anglais on parle des propriétés des chefs (comprenant les forêts et les rivières), la version maorie parle du titre politique de la chefferie. La différence est grande, dans la mesure où ce titre pourrait contrevenir à une pleine et entière souveraineté de la Couronne. L'énumération de ces propriétés est également plus simple en maori : «leurs terres, villages et tous leurs trésors» (Treaty Times Thirty, 2016, p. 40). Il y a une certaine ambiguité dans la version maorie alors que le texte anglais tient à nommer explicitement chaque aspect de cette propriété. Pour ce qui est du droit de préemption, on juge généralement que la traduction rend mal l'appropriation des terres, le terme hokonga pouvant se référer «à l'achat aussi bien qu'à la vente» (Kāwharu, 1989, p. 319; cité dans Schulte-Tenckhoff, 2004, p. 103). Alors que dans la version anglaise, il s'agit d'un droit accordé à la Couronne, on parle en maori de vente, et les sujets du verbe sont les chefs.

Dans le troisième et dernier article, qui fait moins problème, la Couronne confère aux Maoris «tous les droits et privilèges des sujets britanniques» (Treaty Times Thirty, 2016, p. 41) :

\section{Version anglaise datée du 6 février 1840}

In consideration thereof Her Majesty the Queen of England extends to the Natives of New Zealand Her royal protection and imparts to them all the Rights and Privileges of British Subjects.

\section{Traduction maorie de Henry Williams}

Hei wakaritenga mai hoki tenei mo te wakaaetanga ki te Kawanatanga o te Kuini - Ka tiakina e te Kuini o Ingarani nga tangata maori katoa o Nu Tirani ka tukua ki a ratou nga tikanga katoa rite tahi ki ana mea ki nga tangata o Ingarani. 


\section{Rétrotraduction de sir Ian Hugh Kāwharu}

For this agreed arrangement therefore concerning the government of the Queen, the Queen of England will protect all the ordinary people of New Zealand and will give them the same rights and duties of citizenship as the people of England.

La version maorie change quelque peu le sens, d'abord en omettant le concept de sujet, ensuite en garantissant les tikanga, terme employé pour rendre "les droits et privilèges", mais qui réfère plutôt aux "coutumes» (Schulte-Tenckhoff, 2004, p.103). On remarque toutefois que l'équivalent de «the Natives» (tangata maori) peut se traduire par «le peuple» (les Maoris) ou, étymologiquement, «les gens ordinaires» («the ordinary people») ${ }^{17}$.

Le problème du texte ne s'arrête pas à la différence entre deux versions. La conjonction entre ce qui est dit dans le premier et le second article, dans l'une et l'autre langue, soulève aussi des questions : en anglais, le premier article accorde la souveraineté à la Reine, alors qu'en maori, le deuxième article garantit aux chefs la perpétuation de leur autorité. Autrement dit, la primauté politique est accordée, en anglais, au premier article, alors qu'elle l'est, en maori, au second article. Il y a asymétrie non seulement entre les deux langues, mais également entre les concepts. Le révérend Williams aurait-il tenté de tromper les chefs maoris en ne traduisant pas le concept de souveraineté comme il aurait dû le faire? Et comment aurait-il pu traduire ce concept qui, à bien des égards, est un intraduisible de la pensée européenne?

\section{Problèmes de traduction ou de souveraineté?}

La traduction de concepts relevant des sciences humaines et sociales dans un contexte colonial pose un problème particulier, celui de l'incommensurabilité des régimes sémantiques (Santos, 2004, 2007; Price, 2008). Ce problème se constate lorsque l'expérience

17. Les partisans de la version Littlewood interprètent ce passage comme une preuve que le traité éteint définitivement toute forme de droits autochtones en Nouvelle-Zélande, puisque la version Littlewood parle simplement de «the People of New Zealand» (voir Doutré, 2006). Le traité s'adresserait ainsi à tous les Néo-Zélandais, peu importe leur origine. L'interprétation des partisans de la version Littlewood qui ne reconnaissent aucune spécificité aux droits autochtones peut également être remise en question en lisant le préambule du même document, dans lequel Hobson distingue spécifiquement «her Majesty's subjects» des «natives». 
décrite par un concept n'existe pas dans une autre culture. L'enjeu conceptuel devient un problème juridico-politique lorsque l'absence d'un équivalent terminologique est comprise comme un manque dans la culture d'arrivée. Le cas du terme souveraineté est exemplaire, car il suppose l'établissement d'une distribution mondiale d'une seule et même forme politique. Historiquement, on attribue ce manque à un «retard» de la société en question. Ce préjugé reflète ce que Boaventura de Sousa Santos appelle la «pensée abyssale» de l'épistémologie occidentale moderne :

It consists of a system of visible and invisible distinctions, the invisible ones being the foundation of the visible ones. The invisible distinctions are established through radical lines that divide social reality into two realms, the realm of "this side of the line" and the realm of "the other side of the line". The division is such that "the other side of the line" vanishes as reality becomes nonexistent, and is indeed produced as nonexistent. Nonexistent means not existing in any relevant or comprehensible way of being. Whatever is produced as nonexistent is radically excluded because it lies beyond the realm of what the accepted conception of inclusion considers to be its other. What most fundamentally characterizes abyssal thinking is thus the impossibility of the co-presence of the two sides of the line. To the extent that it prevails, this side of the line only prevails by exhausting the field of relevant reality. Beyond it, there is only nonexistence, invisibility, non-dialectical absence. (2007, p. 45-46)

Si le maori ne possède pas de terme pour désigner le concept de souveraineté, on suppose que la raison est à trouver dans le fait que la langue (et la culture qui lui est concomitante) comporte un manque auquel il faut suppléer. Comment dès lors traduire? Isabelle Schulte-Tenckhoff propose deux stratégies (tirées de Biggs, 1989, p. 304-305) :

Lune consiste à assigner une nouvelle signification provenant de la langue-source (en l'occurrence, l'anglais) à un terme déjà existant dans la langue-cible (le maori), dans l'espoir que le terme autochtone ainsi redéfini voudra dire, par décret, ce qu'il doit vouloir dire. [...] [L'autre consiste à créer] de nouveaux termes maoris sur la base d'une translittération de termes anglais [...]. (Schulte-Tenckhoff, 2004, p. 104) 
Cette dernière stratégie a été utilisée par les missionnaires qui ont toujours voulu éviter l'emprunt de termes à connotation magicoreligieuse ${ }^{18}$. Le terme kawanatanga, qui devait rendre souveraineté, est un exemple d'usage de la translittération (de governor) pour éviter d'employer un terme maori en en modifiant le sens. Traduit ainsi, le premier article de la version maorie accorde à la reine le "gouvernorat", un titre politique en second, alors que le deuxième article de la même version accorde la primauté politique aux chefs, à travers le rangatiratanga ${ }^{19}$. En intervertissant la hiérarchie des concepts, le traducteur a-t-il sciemment voulu induire en erreur les signataires maoris, comme le laissent entendre Sabine Fenton et Paul Moon? Faut-il plutôt croire que le traducteur ne possédait pas les compétences nécessaires pour bien traduire les concepts en cause?

Plusieurs commentateurs estiment que, pour que le transfert de la souveraineté soit effectif entre les chefs maoris et l'Empire britannique, Williams aurait dû utiliser un autre terme : mana (Walker, 1989, p. 263; Fenton et Moon, 2002a, p. 55; Fenton et Moon, 2002b, p. 34; Schulte-Tenckhoff, 2004, p. 105). Un des arguments en faveur du terme mana vient de sa présence dans la Déclaration d'indépendance de 1835. La signification de ce terme dans le contexte d'une «souveraineté» européenne est toutefois critiquée par certains, notamment John Laurie :

In fact Williams did not use mana alone but in conjunction with kingitanga to translate the phrase "all sovereign power and authority". [...] I would suggest that, in a word-forword translation, kingitanga signified "sovereignty" and mana "authority". [...] Williams did not use mana in the Treaty of Waitangi to translate "all the rights and powers of sovereignty" because mana is a much vaguer term, which could be used to describe any of the complex bundle of powers and rights exercised by sovereign, government or landowner (and much else as well). (2002, p. 255)

18. Lorsqu'ils traduisent des langues autochtones vers les langues européennes, les anthropologues recourent à la même stratégie, par exemple, en employant le terme mana sans tenter de le traduire.

19. En maori, rangatiratanga désigne la chefferie traditionnelle ou la qualité de chef. Le terme rangatira est assez général pour désigner un supérieur; il s'emploie en maori contemporain pour désigner toute relation hiérarchique, par exemple, celle qui existe entre un employeur et son employé ou entre un propriétaire et son locataire. 
Aucune «solution», aucun «mot juste» ne semble pouvoir rendre intégralement le concept de souveraineté. ${ }^{20}$ Les auteurs qui proposent le terme maori mana désirent simplement faire remarquer que si un transfert de la souveraineté était nécessaire, les signataires du traité devaient en être pleinement conscients. Isabelle Schulte-Tenckhoff va plus loin : elle critique la possibilité même d'un tel transfert en posant de manière particulièrement brillante le paradoxe découlant de l'idée selon laquelle les Maoris ne connaissaient pas le concept de souveraineté parce qu'il s'agissait d'un concept occidental. Voici le paradoxe :

On voit $[\ldots]$ difficilement comment la Nouvelle-Zélande peut nier aux Maoris leur qualité de peuple et, dès lors, leur droit de disposer d'eux-mêmes, tout en affirmant avoir acquis de manière légitime des droits souverains que les Maoris, s'ils n'étaient pas souverains, auraient été incapables de transférer [...]. (Schulte-Tenckhoff, 2004, p. 113)

Dans la perspective politologique de Schulte-Tenckhoff, si les Maoris possédaient une souveraineté avant la signature du traité de Waitangi, le traité ne pouvait se situer qu'entre deux parties souveraines. L'interprétation du traité ne peut alors être débattue que dans une cour de justice internationale, entre deux États souverains. Cette conséquence exclut toutefois qu'une des parties ne soit pas souveraine et rend ainsi caduc le transfert de la souveraineté. Le statut du traité qui, actuellement, est interprété par la cour néo-zélandaise (ce que Schulte-Tenckhoff appelle l'«internalisation $\left.{ }^{21}\right)$, semble indiquer que, du point de vue de l'Empire, il n'y a jamais eu de souveraineté maorie, et donc qu'elle n'a jamais pu être transférée. Dans un cas comme dans l'autre, la souveraineté ne se transmet pas comme elle ne se traduit pas.

Le titre de l'article de Schulte-Tenckhoff (2004) proposait une option à laquelle il vaut la peine de réfléchir: "Te tino rangatiratanga: substance ou apparence?». Schulte-Tenckhoff n'explique jamais ces deux termes, sauf lorsqu'elle cite un chef maori signataire, le chef Nopera, qui commente le traité : "Seule l'ombre de la terre a été transférée à la Reine, tandis que le sol

20. Le terme Kingitanga n'est pas non plus une solution idéale, car il s'agit encore une fois d'une translittération, celle du mot anglais King à laquelle le suffixe -tanga a été ajouté (en 1835, Guillaume IV, prédécesseur de Victoria, est le souverain de la Grande-Bretagne).

21. En anglais, Schulte-Tenckhoff (1998) parle de «domestication». 
nous est resté» (cité dans Ngata, 1922, p. 10; voir aussi SchulteTenckhoff, 2004, p. 113, qui ajoute entre crochets, après le mot sol, «[ou : la substance]»). L'ombre désigne ici la «souveraineté» (ou le kawanatanga, du point de vue maori), la représentation ou la vicariance (c'est bien un vice-roi représentant de la Reine qui sera nommé à la suite de la signature du traité). On pourrait aussi l'interpréter comme le "concept», qui s'opposerait à la «chose en soi», au sol ou à la terre, à la chefferie ou au rangatiratanga. Le chef Nopera commencera à douter du bien-fondé du traité peu de temps après sa signature, dès janvier 1841, en indiquant que, peut-être, c'est l'ombre que les chefs conserveront (Ross, 1972, p. 142-143; cité dans Dawson, 2005, p. 48). Dans un cas comme dans l'autre, on pense la souveraineté dans une ontologie à valence unique : être ou ne pas être, jamais les deux à la fois ${ }^{22}$.

On peut toutefois comprendre autrement la souveraineté, ce qui nous oblige à repenser la traduction : elle est une performance de l'État. La souveraineté se caractériserait ainsi comme une propriété émergente de la force de l'État. Cette performance serait donc l'acte toujours à refaire de l'auto-affirmation de l'État en tant qu'État (sur cette interprétation du concept de souveraineté, voir Giroux, 2014). Refuser de reconnaître la souveraineté du peuple maori au moment où on prétend qu'elle est transférée à l'Empire colonial ne serait donc pas un paradoxe, mais plutôt la condition même de la souveraineté coloniale de se constituer dans le refus de la souveraineté de l'autre colonisé. Pour nier tout droit au lieu, il ne s'agit toutefois pas de constater simplement son absence concrète - comme le fait Locke à partir de son Angleterre sans jamais mettre les pieds en Amérique -, il faut simuler l'absence de droit (terra nullius) par un discours, celui de la découverte d'un «Nouveau Monde» (doctrine de la découverte) ${ }^{23}$.

Comment dès lors «bien» traduire un document comme le traité de Waitangi? Contrairement aux interprétations dominantes concernant la traduction effectuée par Henry

22. Avant Shakespeare, il y a eu Aristote: «Il est impossible que le même simultanément appartienne et n'appartienne pas au même et selon le même.» (Aristote, au livre Gamma, 1005 b 19-20; cité dans Cassin, 2010, §31).

23. Simon Labrecque (2017) analyse dans une perspective traductologique les rapports entre la politique de la colonisation et sa traduction dans le langage du droit, particulièrement chez Carl Schmitt (Labrecque cite principalement Schmitt, 2012). 
Williams - selon lesquelles il aurait mal traduit le texte, en raison de son incompétence ou à dessein -, je proposerais de considérer que le révérend Williams a très bien traduit le texte. Cependant, pour adopter un tel point de vue, il faut repenser la traduction. Dans le premier paragraphe du traité, un concept étranger, la souveraineté, est translittéré: Williams le rend avec un terme étranger (kawanatanga est un terme étranger pour un concept étranger). Dans le deuxième paragraphe, pour parler du pouvoir que les chefs continueront d'exercer, Williams le rend simplement par le terme que les signataires connaissaient: rangatiratanga. Ce n'est pas tant la hiérarchie entre les concepts qui compte que leur distribution : Williams rend pluriels les lieux du pouvoir et il multiplie les interprétations possibles; il empêche la détermination d'un centre et contrecarre l'unicité d'un lieu de décision. Prendre au sérieux la plurivocité des sens du traité de Waitangi, c'est obliger à penser plus d'une souveraineté et, par conséquent, sa ruine possible. Williams nous force à penser la pluralité des lieux de pouvoir en nous obligeant à interpréter la pluralité du sens des différentes versions du traité. Sans peut-être le savoir ou le vouloir, Williams offrait une nouvelle image politique de la traduction en traduisant contre l'État.

\section{Conclusion}

Et cette pensée sauvage, presque aveuglante de trop de lumière, nous dit que le lieu de naissance du Mal, de la source du malheur, c'est l'Un.

(Clastres, 1974, p. 184).

Le traité de Waitangi comme texte multiple pose le double problème d'une politique de la traduction et de la traduction de la politique. La question juridico-politique des «deux traités» est, en définitive, celle de savoir si les Maoris ont aliéné leur souveraineté. Or, la question qu'on pourrait se poser à partir d'une réflexion traductologique est la suivante : pour pouvoir traduire le concept de souveraineté, ce concept doit-il déjà exister en maori? Ce concept, compris comme l'unité des relations entre le territoire, le peuple et sa représentation dans un corps politique, peut-il être pensé à partir d'un signifié transcendantal qui permettrait une équivalence formelle en traduction interlinguistique (Jakobson, 1986)? J'ai tenté de penser différemment le problème : et si la souveraineté 
comme concept était la marque laissée d'une «fixion» (Cassin, 2010, §1)? ${ }^{24}$ Cette fixion - à la fois fixation et fiction - serait celle d'une équivalence formelle entre les langues et les cultures, elle performerait la reconnaissance d'une égalité tout en la niant. La traduction comme transfert devient possible et pensable, mais aux conditions de sa propre ruine. Elle se performe si, et seulement si, elle nie à l'autre ses attributs singuliers. C'est là une aporie de la politique coloniale, et cette aporie est directement liée à la pensée dominante de la traduction comme transfert inentamable de l'univocité du sens.

L'histoire de la traduction du traité de Waitangi montre que l'origine n'est jamais unique ou singulière; elle est bien souvent elle-même l'être en second, posée a posteriori pour répondre à des exigences judicatives, celle d'une critique qui pourrait poser un regard désapprobateur sur les "problèmes» de traduction. La particularité de l'histoire de la traduction du traité de Waitangi est peut-être finalement que sa fonction même de traduction n'est pas si évidente. La version maorie, parce que c'est elle qui a été signée, s'institue comme origine, et la chance que la traduction de Williams apporte est de multiplier les lieux interprétatifs. La question de savoir si Williams a bien ou mal traduit devient moins importante que celle de savoir quels sont les effets d'une telle traduction dans un contexte colonial. N'y a-t-il pas lieu de repenser la nécessité de faire intervenir plus d'une interprétation légitime des textes, une certaine relativité dans la constitution du sens et une vulnérabilité dans son rapport à l'autre?

Henry Williams, ce traducteur tant décrié, nous force peutêtre à repenser la conception mécanique de la traduction comme univocité du sens - une seule interprétation possible - et comme transfert du message - une équivalence entre deux versions. Le transfert est une métaphore de la traduction qui n'est ni naturelle ni universelle (Simeoni, 2014) : elle est une construction philosophique dont on peut retracer les racines dans la philosophie antique. Une autre pensée de la traduction est-elle possible? Contre un universalisme de la langue en traduction, contre une pensée de la langue comme simple moyen de communication du sens univoque, la philosophe Barbara Cassin propose de revenir à un usage sophistique de la langue et à une logique relativiste :

24. Chez Cassin, l'usage du «x lacanien» sert à «souligner que [c'] est une fabrication, le factum un fictum que l'on décide de fixer» $(2010, \S 1)$. 
[C] que je propose d'appeler le comparatif dédié : «meilleur pour». Le meilleur est en effet défini comme «le plus utile», le mieux adapté à (la personne, la situation, toutes les composantes de ce moment que les Grecs nomment kairos, "opportunité»). [...] Dans cette perspective, il n'y a pas d'être à chercher sous l'apparaître («est-et-apparaît» fait syntagme), pas d'Un à chercher sous ou au-dessus du multiple. Il n'y a pas de point de vue de Dieu, comme chez Leibniz, pour unifier toutes les perceptions des monades. $(2010, \S 41-42)$

C'est peut-être là l'enseignement à retenir de l'histoire du traité de Waitangi si on veut décoloniser la traduction : comment assurer à la traduction une plurivocité qui ne scelle pas l'interprétation? Quel kairos une «mauvaise» traduction peut-elle offrir?25

\section{Références}

Aborigines' Protection Society (1840). Outline of a system of legislation for the securing of protection to the Aboriginal inhabitants of all countries colonized by Great Britain: Extending to the political and social rights, ameliorating their condition, and promoting their civilization. London, John Murray.

Anker, Kirsten (2014a). Declarations of Interdependence: A Legal Pluralist Approach to Indigenous Rights. New York, Routledge.

Anker, Kirsten (2014b). «Translating Sui Generis Aboriginal Rights in the Civilian Imagination». In A. Popovici, L. Smith et R. Tremblay, dir. Les intraduisibles en droit civil. Montréal, Éditions Thémis, p. 1-36.

Asch, Michael (2014). On Being Here to Stay: Treaties and Aboriginal Rights in Canada. Toronto, University of Toronto Press.

Bennett, Karen (2007). «Epistemicide! The Tale of a Predatory Discourse». The Translator, 13, 2, p. 151-169.

Biggs, Bruce (1989). «Humpty-Dumpty and the Treaty of Waitangi». In I. H. Kāwharu, dir. Waitangi: Māori and Päkehā Perspectives of the Treaty of Waitangi. Auckland, Oxford University Press, p. 300-312.

Bodin, Jean (1993 [1583]). Les six livres de la République. Paris, Librairie générale française.

25. Avec la résurgence du droit autochtone au Canada et dans d'autres pays de tradition britannique, on observe une réinvention tout à fait originale des formes de vie possibles dans une société en contexte colonial (je pense à Borrows, 2010; Anker, 2014a; Asch, 2014). Il y a lieu de se demander ce que les traductologues peuvent offrir comme réflexion sur les relations langagières dans ce contexte. Cet article se voulait un appel plutôt qu'une réponse définitive à cette problématique. 
Borrows, John (2010). Canada's Indigenous Constitution. Toronto, University of Toronto Press.

Burger, Peter (2011). Waitangi: What Really Happened. Eyeworks New Zealand, $68 \mathrm{~min}$.

Cassin, Barbara (2010). «Relativité de la traduction et relativisme». In A. Berthoz, C. Ossola et B. Stock, dir. La Pluralité interprétative. Fondements historiques et cognitifs de la notion de point de vue. Paris, Collège de France. Disponible à : <http://books.openedition.org/ cdf/1449> [consulté le 2 juin 2017].

Cheyfitz, Eric (1997). The Poetics of Imperialism: Translation and Colonization from the Tempest to Tarzan. Philadelphie, University of Pennsylvania Press.

Clastres, Pierre (1974). La société contre l'État. Paris, Éditions de Minuit. Court of Appeal of New Zealand (1987). New Zealand Maori Council v Attorney-General, 1 NZLR 641.

Dawson, Richard (2005). «Waitangi, Translation, and Metaphor». Site: A Journal of Social Anthropology and Cultural Studies, 2, 2, p. 33-63.

Doutré, Martin (2005). The Littlewood Treaty: The True English Text of the Treaty of Waitangi Found. Auckland, De Danaan Publishers.

Doutré, Martin (2006). «Martin Doutrés Response to Dr. Donald Loveridge's Article». 14 juillet. Disponible à : <http://www. treatyofwaitangi.net.nz/LoveridgeResponse.htm> [consulté le 2 mai 2017].

Fenton, Sabine et Paul Moon (2002a). «Bound Into a Fateful Union: Henry Williams'Translation of the Treaty of Waitangi Into Maori in February 1840». The Journal of the Polynesian Society, 111, 1, p. 51-63.

Fenton, Sabine et Paul Moon (2002b). «The Translation of the Treaty of Waitangi : A Case of Disempowerment». In M. Tymoczko et E. Gentzler, dir. Translation and Power. Boston, University of Massachusetts Press, p. 25-44.

Geslin, Albane (2015). «Plurilinguisme, traduction et droit international des peuples autochtones: en quête de reconnaissance». Sens public, «Langues \& Normes». Disponible à : <http://www.sens-public.org/ article1139.html> [consulté le 30 juin 2017].

Giroux, Dalie (2014). «Comment fabriquer un État en Amérique, ou : la Vierge, le Diable, le Boucher et Carcajou ». Les Cabiers de l'imaginaire, 12, p. 68-89.

Hegel, Georg Wilhelm Friedrich (1965). La raison dans l'Histoire: introduction à la Philosophie de l'Histoire. Trad. Kostas Papaioannou. Paris, Librairie Plon.

Henderson, James (Sákéj) Youngblood (2000). «The Context of the State of Nature». In M. Battiste, dir. Reclaiming Indigenous Voice and Vision. 
Vancouver, University of British Columbia Press, p. 11-38.

Hobbes, Thomas (2002 [1651]). Léviathan. Traité de la matière, de la forme et du pouvoir de la république ecclésiastique et civile. Trad.Philippe Folliot. Disponible à : <http://classiques.uqac.ca/classiques/hobbes_ thomas/leviathan/leviathan.html> [consulté le 15 juin 2017].

Jakobson, Roman (1986). «Aspects linguistiques de la traduction». Essais de linguistique générale. Paris, Éditions de Minuit, p. 78-86.

Kāwharu, Ian Hugh (1989). "Translation of the Treaty of Waitangi». In I. H. Kāwharu, dir. Waitangi: Mãori and Päkehà Perspectives of the Treaty of Waitangi. Auckland, Oxford University Press, p. 319-321.

Labrecque, Simon (2017). "La découverte de l'Amérique». Trabir, 8. Disponible à : <https://trahir.wordpress.com/2017/05/24/labrecquedecouverte/> [consulté le 15 septembre 2017].

Laurie, John (2002). «Translating the Treaty of Waitangi ». The Journal of the Polynesian Society, 111, 3, p. 255-258.

Lemieux, René (2014). «La traduction à l'époque de sa reproductibilité technique. Ou, L'impossible dissonance interculturelle». In E. Diamanti, M. Rosales et D. Zawadzka, dir. Les dissonances du vivoreensemble. Actes du colloque étudiant du CELAT. Montréal, CELAT, p. 19-30. Disponible à : <http://www.celat.ulaval.ca/wp-content/ uploads/2014/05/Actes-comp1\%C3\%A8tes-Les-Dissonances-duvivre-ensemble-r\%C3\%A9duit.pdf\#page=21> [consulté le 30 juin 2017].

Liu, Lydia H. (1995). Translingual Practice: Literature, National Culture, and Translated Modernity - China, 1900-1937. Stanford, Stanford University Press.

Locke, John (1992 [1795]). Traité du gouvernement civil. Trad. David Mazel. Disponible à : <http://classiques.uqac.ca/classiques/locke_ john/traite_du_gouvernement/traite_du_gouver_civil.html $>$ [consulté le 30 juin 2017].

Loveridge, Donald (2006). The "Littlewood Treaty»: An Appraisal of Texts and Interpretations. Treaty of Waitangi Research Unit. Disponible à : <https://www.victoria.ac.nz/stout-centre/research-units/towru/ publications/The-Littlewood-Treaty.pdf> [consulté le 2 mai 2017].

Morin, Michel (1997). L'Usurpation de la souveraineté autochtone. Le cas des peuples de la Nouvelle-France et des colonies anglaises de l'Amérique du Nord. Montréal, Éditions du Boréal.

Nairn, Mitzi (2013). «Colonial Myths: "He i wi kotabi tātou"». The Treaty People Blog: Articles, reports, and journals from Treaty Workers in Aotearoa. 11 octobre. Disponible à : <https://treatypeople.wordpress. com/2013/10/11/colonial-myths-he-iwi-kotahi-tatou/> [consulté le 30 juin 2017]. 
New Zealand Parliament/Pāremata Aotearoa (1975). Treaty of Waitangi Act 1975, 1975 No 114. Disponible à : <http://www.legislation.govt. nz/act/public/1975/0114/92.0/DLM435368.html> [consulté le 2 mai 2017].

Ngata, Apinara (1922). The Treaty of Waitangi: An Explanation. Trad. Michael R. Jones. Maori Purposes Fund Board. Disponible à : $<$ http://nzetc.victoria.ac.nz/tm/scholarly/tei-NgaTrea-t1-g1-t1. html> [consulté le 2 mai 2017].

Normanby, C. H. P. (1990 [1839]). «The Marquis of Normanby to Captain Hobson, R.N. No. 6.» In A. Mackay, A Compendium of Official Documents Relative to Native Affairs in the South Island, Volume One [1873]. Wellington, The Alexander Turnbull Library. Disponible à : $\quad<$ http://nzetc.victoria.ac.nz/tm/scholarly/tei-Mac01Comp-t1g1-t5-g1-t2-g1-t6.html> [consulté le 2 mai 2017].

Orange, Claudia (2010 [1987]). The Treaty of Waitangi. Wellington, Bridget Williams Books.

Parent, Arnaud (2009). «On Ambiguity and Incongruity of Terms in Translations of International Treaties». Vertimo Studijos, 2, p. 144161.

Parkinson, Phil G. (2006). "Preserved in the Archives of the Colony": The English Drafts of the Treaty of Waitangi». Revue juridique polynésienne, cahier spécial. New Zealand Association for Comparative Law.

Price, Joshua M. (2008). «Translating Social Science: Good versus Bad Utopianism ». Target, 20, 2, p. 348-364.

Price, Joshua M. et María Lugones (2003). «Problems of Translation in Postcolonial Thinking». Anthropology News, 44, 4, p. 7-9.

Rogers, Lawrence M. (1973). Te Wiremu: A Biography of Henry Williams. [Christchurch, N.Z.], Pegasus Press.

Ross, Ruth (1972). «Te Tiriti o Waitangi: Texts and Translations». New Zealand Journal of History, 6, 2, p. 129-157.

Santos, Boaventura de Sousa (2004). "A Critique of Lazy Reason: Against the Waste of Experience». In I. Wallerstein, dir. The Modern World-System in the Longue Durée. Boulder, Paradigm Publishers, p. 157-197.

Santos, Boaventura de Sousa (2007). «Beyond Abyssal Thinking: From Global Lines to Ecologies of Knowledges». Revista Critica de Ciências Sociais, 77. Disponible à : <http://www.eurozine.com/ beyond-abyssal-thinking/> [consulté le 2 mai 2017].

Schmitt, Carl (2012). Le nomos de la Terre. Dans le droit des gens du Jus Publicum Europaeum. Trad. Lilyane Deroche-Gurcel. Paris, Presses Universitaires de France. 
Schulte-Tenckhoff, Isabelle (1998). "Reassessing the Paradigm of Domestication: The Problematic of Indigenous Treaties ». Review of Constitutional Studies, IV, 2, p. 239-289.

Schulte-Tenckhoff, Isabelle (2004). «Te tino rangatiratanga: substance ou apparence? Réflexion sur le dilemme constitutionnel de l'État néo-zélandais ». Politique et Sociétés, 23, 1, p. 89-114.

Sharp, Andrew (1990). Justice and the Mãori: Māori Claims in New Zealand Political Argument in the 1980s. Auckland, Oxford University Press.

Simeoni, Daniel (2014). «De quelques usages du concept de transfert dans la réflexion sur la traduction ». In P. Gin, N. Goyer et W. Moser, dir. Transfert. Exploration d'un champ conceptuel. Ottawa, Presses de l'Université d'Ottawa, p. 103-117.

Supreme Court of New Zealand (1877). Wi Parata v Bishop of Wellington. Te Paipera Tapu 1868: The Holy Bible in Maori (2013 [1868]). Trad. Henry Williams, Robert Maunsell et William Williams. Auckland, Trinitarian Bible Society of New Zealand.

The Treaty Times Thirty: Translating the Treaty of Waitangi into Thirty Languages/Te Tiriti Whakareatia ki te Toru Tekau (2016). Auckland, The New Zealand Society of Translators and Interpreters. Disponible à : <https://treatytimes30.org/> [consulté le 2 mai 2017].

Walker, Ranginui J. (1989). "The Treaty of Waitangi as the Focus of Maori Protest». In I. H. Kāwharu, dir. Waitangi: Maori and Pakeha Perspectives of the Treaty of Waitangi. Auckland, Oxford University Press, p. 263-279.

René Lemieux

Département des lettres et communication Université de Sherbrooke 2500, boul. de l'Université Sherbrooke (Québec) J1K 2R1 Rene.Lemieux@USherbrooke.ca 